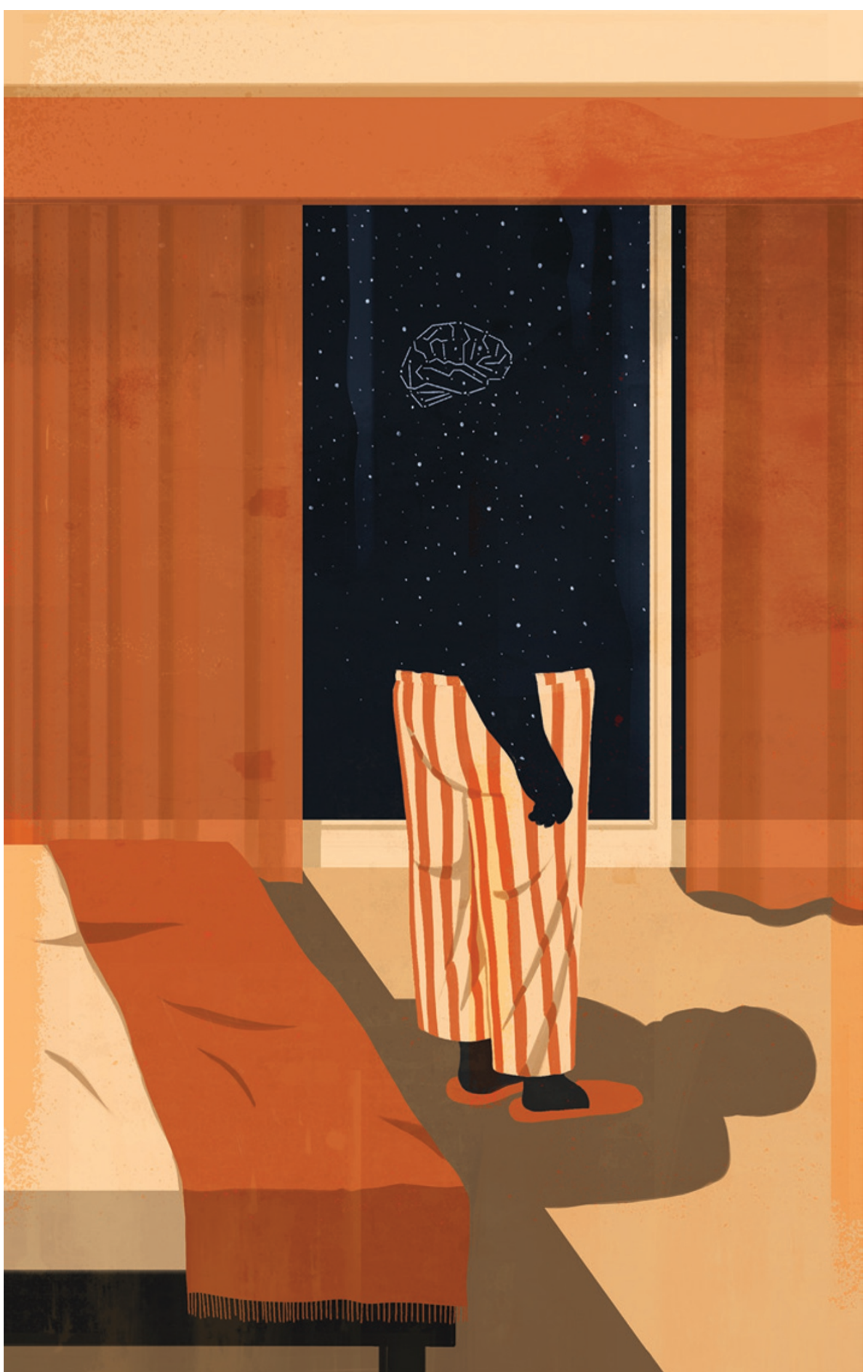

OBESITY

\section{Heavy sleepers}

\section{A growing body of evidence shows that getting a good night's sleep plays an important role in regulating the body's metabolism.}

\section{BY BRIAN OWENS}

$\mathrm{B}$ urning the midnight oil can leave you tired and grumpy the next day, dulling your mind and slowing your reaction times. But lack of sleep has consequences beyond the brain as well, with long-term sleep disturbances leading to metabolic problems.

Matthew Brady, a biologist at the University of Chicago in Illinois who studies the links between sleep and metabolism, puts it simply: "Fat cells need their sleep as well."

Many large population studies over the past decade have found that people who sleep poorly are more likely to be obese and to have metabolic disorders such as type 2 diabetes. One study of more than 1,000 people found that sleeping for only 5 hours rather than 8 hours results in a 3.6\% increase in body mass index.

There is evidence that it's this lack of sleep that causes metabolic disorders and weight gain. In 1999, Eve Van Cauter and her colleagues at the University of Chicago showed that sleep restriction in healthy young men led to signs of insulin resistance, which can lead to type 2 diabetes. This finding "changed the entire world of sleep" research, says Fred Turek, who studies the biology of circadian rhythms at Northwestern University in Evanston, Illinois. Before Van Cauter's research, the only known effect of a lack of sleep was that "you were tired", Turek says, but the study revealed that "people getting too little sleep are at risk of obesity, diabetes and other metabolic disorders."

The underlying biology is slowly becoming clear. Van Cauter's work helped to show that two hormones - leptin and ghrelin - are likely to be involved in the link between sleep and weight gain. Leptin is produced by fat cells and is a signal of satiety; ghrelin is produced by the stomach and signals hunger. Together, these hormones regulate hunger and appetite. Van Cauter's team later showed that restricting the sleep time of healthy young men in the lab caused their leptin levels to fall and their ghrelin levels to rise, increasing their appetite, especially for fatty and sugary foods ${ }^{1}$.

The biochemical pathway that leads from lack of sleep to changes in leptin and ghrelin levels is still being investigated, says Esra Tasali of the University of Chicago, who worked with Van Cauter on the leptin-ghrelin study. Tantalizing clues emerged from a study published in 2012 by Tasali, Brady and Van Cauter showing that reduced sleep leads to increased insulin resistance in fat cells. The effect was huge: after four nights of just 4.5 hours of sleep, the fat cells of young, healthy volunteers showed a $30 \%$ reduction in insulin sensitivity ${ }^{2}$. That's "the equivalent of metabolically ageing them 10 to 20 years", says Brady.

So reducing sleep can have a profound effect on individual cells. "The fat cell's getting some signal saying 'I'm not sleeping much', and that's going to alter its biology," says Turek.

Metabolically, sleep quality is just as important as sleep duration. The onset of slow-wave sleep coincides with hormonal changes that 
affect glucose regulation, such as the release of growth hormone. When people are allowed to sleep for 7 or 8 hours, but are prevented for several consecutive nights from going into deep, slow-wave sleep, they show the same insulin signalling response as if they had been sleeping for only 4 hours.

The link between sleep and metabolism might be controlled by a part of the autonomic nervous system called the sympathetic nervous system, says Tasali. Activity of the sympathetic nervous system inhibits digestion, and so suppresses the secretion of insulin from the pancreas. Brady adds that because leptin levels are proportional to insulin-stimulated glucose uptake in fat cells, a reduction in insulin sensitivity could lead to less leptin production, which would in turn stimulate appetite and potentially lead to weight gain.

\section{WE'VE GOT RHYTHM}

The interaction between sleep and metabolism is complicated by the poorly understood relationship between sleep and the body's natural circadian rhythms. "If you disrupt circadian rhythmicity, you're going to have effects on the sleep-wake cycle," says Turek. "And if you disrupt the sleep-wake cycle, you're probably having effects on various circadian rhythms as well."

The circadian rhythm is determined by a collection of interrelated biochemical clocks that influence when we sleep, when we eat, and many other biological activities (see 'Stepping out of time', page S10). Mice that lack a working copy of a protein called Clock develop high blood sugar and cholesterol levels and low insulin, eventually becoming obese ${ }^{3}$.

The Clock protein is active in a brain region known as the suprachiasmatic nucleus (SCN), which is linked to the light-dark cycle of day and night. The SCN synchronizes all the body's 24-hour clocks, either directly, for example by stimulating the pineal gland to produce the hormone melatonin, or indirectly, by influencing the time of feeding, which activates pancreatic function. But, says Turek, "humans are the only species that doesn't pay attention to their biological clock" - we eat whenever we want.

To study the metabolic effect of eating at different times, Turek altered the natural feeding time of mice. He fed one group of mice a high-fat diet only during the day, when these nocturnal animals would not normally be eating, and fed another group the same diet only at their biologically normal time, at night. The results sound a cautionary note to those of us who consistently fall out of sync with our body's natural eating schedule. Although both groups of mice consumed the same amount

$\rightarrow$ NATURE.COM For more on the link between sleep and metabolism: go.nature.com/wrp4ku more body fat than those fed at night. "Eating at the wrong time of day leads to alterations in something - maybe basal metabolic rate, maybe body temperature - that changes how the body processes the energy that it takes in," he says. "If you're eating at the wrong time of day, you're more likely to gain weight."

So when humans override the SCN's signal by eating late at night, they throw their feeding cycle and various downstream rhythms out of alignment with the central nervous system, with possible knock-on consequences for their metabolism. "It's not only what you eat, it's when you eat," says Turek.

\section{SLEEP IT OFF}

It's clear then that bad or shortened sleep causes metabolic problems. But would getting more and better sleep reverse these effects for example, by helping obese people to lose weight? Research by Tasali and others suggests that such therapeutic effects are possible.

Tasali has focused on improving sleep quality in pre-diabetic patients who suffer from sleep

"If you're eating
at the wrong
time of day,
you're more
likely to gain
weight."
apnoea, a breathing disorder that leads to disturbed sleep. "We can give them back their sleep quality by treating their sleep apnoea, and see what happens to their glucose metabolism," she says. In a study presented at the American Thoracic Society meeting in Philadelphia, she found that a two-week regimen of the standard treatment for sleep apnoea, called continuous positive airway pressure, led to greatly reduced insulin resistance ${ }^{4}$.

Similarly encouraging results have come from weight-loss research. Obesity specialist Giovanni Cizza at the National Institute of Diabetes and Digestive and Kidney Diseases in Bethesda, Maryland, has been testing whether overweight people who are short sleepers can lose weight if they are coached to sleep longer ${ }^{5}$. The study ended in 2012 and Cizza is now analysing the data. He says he is "pleased" with the results, despite a major hiccup in the trial.

The subjects were split into an intervention group, in which subjects were coached about good sleep hygiene and how to improve their sleep habits, and a comparison group, given no coaching. But simply making the comparison group more aware of their sleep habits, by asking them to fill out sleep diaries, helped them extend their sleep duration by almost as much as the intervention group. Basically, Cizza says, "my placebo group took the drug too". The results have not yet been published, so he would not say how much weight the subjects lost - only that because both groups extended their sleep, there was no significant difference between them in sleep duration or weight loss.

Clearly, the relationship between sleep and metabolism is complicated. A host of interacting biological, behavioural and psychological factors influence both sleep and metabolic function, says Jim Horne, a sleep researcher at Loughborough University, UK. People who sleep less have more time to spend eating, for example. The stress and tiredness caused by a bad night's sleep can lead to comfort eating and less activity during the day. In addition, obesity is known to cause breathing problems, such as sleep apnoea, that can interrupt sleep. And both poor sleep and obesity are often associated with other disorders, such as depression (see 'The dark night', page S14).

As a result, it is unclear whether short sleep duration causes obesity, or vice versa, says Horne. "I challenge this idea that short sleep makes you fat. I would say being fat causes short sleep and bad sleep."

But it may not be necessary to untangle cause and effect fully before testing the simple proposition of whether better sleep can help people lose weight. Because sleep is not a drug, testing its effects should be a less daunting process than that required for pharmaceutical development, says Cizza. After all, he points out, if you're not getting enough sleep, getting more will have no adverse side effects, and will have many added benefits in terms of mood and neuropsychological function, for example. "Where there is reasonable evidence for efficacy and there is no harm, I think we should bring this to the public sooner than a drug."

Brady points out that people often find it difficult to change their diet and take more exercise. "Telling them to sleep more, we think that might be a little more palatable."

Horne, however, urges caution. In most cases, he says, the effect of extra sleep on metabolism is vanishingly small. "Fifteen minutes of brisk walking every day is far better at regulating your body weight than an extra hour of sleep, so we have to put things in perspective." He worries that people will be tempted to avoid healthy exercise in favour of the less strenuous option of popping sleeping pills, which do have side effects. That could be dangerous, and no one working on the complex relationship between sleep and metabolism would recommend trying such a shortcut.

But there is mounting evidence that getting the right amount of healthy sleep can be just as important as diet and exercise in controlling your metabolism, and can do a world of good beyond making you wake up happy in the morning.

Brian Owens is a freelance writer based in St Stephen, New Brunswick, Canada.

1. Siegel, K. et al. Ann. Intern. Med. 141, 846-850 (2004).

2. Broussard, J. et al. Ann. Intern. Med. 157, 549-557 (2012).

3. Turek, F. et al. Science 308, 1043-1045 (2005).

4. Pamidi, S. et al. American Thoracic Society Meeting, 20 May 2013

5. Cizza, G. et al. Clin. Trials. 7, 274-285 (2010). 